Artículo

\title{
Relación entre la resistencia a antibióticos y la producción de biofilm de aislados de Staphylococcus aureus provenientes de mastitis bovina
}

Jaquelina Julia Guzmán-Rodríguez ${ }^{\text {a,b }}$

Estefanía Salinas-Pérez ${ }^{\text {b }}$

Fabiola León-Galván ${ }^{\text {a,c }}$

José Eleazar Barboza-Corona ${ }^{\text {a,c }}$

Mauricio Valencia-Posadas ${ }^{\mathrm{a}, \mathrm{b}}$

Fidel Ávila-Ramos a,b

José Antonio Hernández-Marín ${ }^{a, b}$

Diana Ramírez-Sáenz ${ }^{\mathrm{d}}$

Abner Josué Gutiérrez-Chávez ${ }^{\text {a,b* }}$

${ }^{a}$ Universidad de Guanajuato. Campus Irapuato-Salamanca. División de Ciencias de la Vida, Programa de Posgrado en Biociencias. Km. 9.0 Carr. Irapuato-Silao, El Copal, Irapuato, 36821, Guanajuato, México.

${ }^{\mathrm{b}}$ Universidad de Guanajuato. Campus Irapuato-Salamanca. División de Ciencias de la Vida, Departamento de Medicina Veterinaria y Zootecnia.

c.Universidad de Guanajuato. Campus Irapuato-Salamanca. División de Ciencias de la Vida, Departamento de Alimentos. México.

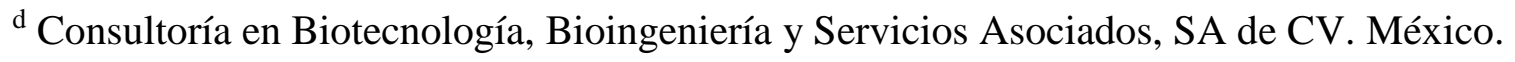

*Autor de correspondencia: ajgutierrez@ugto.mx

\section{Resumen:}

El objetivo fue analizar la relación entre el perfil de resistencia a antibióticos y la formación de biofilm de aislados de $S$. aureus provenientes de mastitis bovina. Se analizaron 30 aislados 
de $S$. aureus procedentes de casos de mastitis subclínica en granjas lecheras en sistemas de producción semi-intensivo y de traspatio ubicadas en los estados de Guanajuato y Michoacán, México. Se realizó un antibiograma por el método de difusión en disco Kirbi Bauer. La formación de biofilm se determinó por el método de tinción con cristal violeta. Para la evaluación de genes de resistencia a antibióticos y de formación de biofilm se obtuvo ADN genómico de una colonia para la identificación de los genes: blaZ, mecA, tetK, tetM, gyrA y gyrB, y icaA e icaD. Los resultados mostraron que el $100 \%$ de los aislados fueron resistentes a penicilina y dicloxacilina, seguidos por cefotaxima (86.6\%), ampicilina y cefalotina $(83.3 \%)$ y ceftazidima $(80.0 \%)$, mientras que se observó un $36.6 \%$ de resistencia a oxacilina. Se identificó que todos los aislados de $S$. aureus presentaron la capacidad de formar biofilm con un rango del 20 a $98 \%$. Se observó además que los aislados con una multirresistencia elevada presentaron una mayor formación de biofilm; estableciéndose una correlación positiva significativa. En conclusión, los aislados de $S$. aureus provenientes de mastitis bovina presentaron elevados niveles de resistencia a antibióticos; así como una importante capacidad formadora de biofilm, demostrando la existencia de una correlación positiva entre estos dos factores.

Palabras clave: Antibióticos, Mastitis, ADN, Biofilm.

Recibido: 20/03/2020

Aceptado: 12/03/2021

\section{Introducción}

El procesamiento de la leche bovina es un sector de suma importancia en la industria ganadera, en México, se estimó una producción superior a los 12 millones de toneladas en el $2019^{(1)}$, lo que lo ubica dentro de los primeros diez países productores de leche a nivel mundial ${ }^{(1)}$. Uno de los principales objetivos de una granja lechera debería ser tener una producción eficiente de leche y que ésta sea saludable y libre de contaminantes, por lo que es esencial que la glándula mamaria esté saludable ${ }^{(2)}$. En este sentido, la mastitis es la enfermedad más común y costosa en el ganado bovino lechero, ya que afecta el bienestar de la vaca y causa problemas económicos por pérdidas en producción, disminución de calidad y cantidad de leche, eliminación prematura de la vaca, costo de tratamiento veterinario y el descarte de la leche debido a la contaminación con antibiótico ${ }^{(3,4)}$.

Staphylococcus aureus es un patógeno ubicuo que causa una variedad de infecciones en humanos y animales y es uno de los principales agentes causales de la mastitis bovina ${ }^{(5,6)}$. Esta bacteria Grampositiva produce infecciones crónicas, persistentes y recurrentes, ya que es capaz de superar todas las barreras del sistema de defensa del huésped, debido a que posee un amplio espectro de factores de virulencia como la producción de enzimas, antígenos, 
adhesinas y toxinas, entre otros ${ }^{(7)}$. Estos factores de virulencia eventualmente le confieren a la bacteria la multirresistencia a los antibióticos y la formación de biopelículas ("biofilm")(8). El biofilm es un consorcio de microorganismos que se encuentra embebido dentro de una matriz polimérica, constituida principalmente por exopolisacaridos, proteínas y ácidos nucleicos, que le permite a la bacteria adherirse a una superficie biótica o abiótica ${ }^{(9)}$. La formación de biofilm es una estrategia de vida para la mayoría de las bacterias, ya que ésta les brinda estabilidad, desempeña funciones catalíticas, aumenta las posibilidades de transferencia de material genético y la resistencia a los antibióticos, participa en los procesos de comunicación celular y ofrece protección para sobrevivir a las condiciones adversas y variables del ambiente; lo que contribuye a su colonización exitosa en el hospedador ${ }^{(10)}$. La multirresistencia a antibióticos y la formación de biofilms son características de virulencia que se encuentran relacionadas entre sí de manera importante. En este sentido, se sabe que el biofilm formado por $S$. aureus aumenta significativamente la resistencia a los antibióticos, al inhibir la penetración del antimicrobiano lo que resulta en una situación cada vez más grave en el combate terapéutico de este microorganismo ${ }^{(11)}$.

Actualmente nuestro grupo cuenta con una colección de aislados de $S$. aureus provenientes de mastitis bovina que fueron colectados en los estados de Guanajuato y Michoacán, México, los cuales han presentado niveles de multirresistencia a antibióticos muy elevados (70 a $100 \%)^{(12)}$, lo que es congruente con la poca eficiencia en las terapias utilizadas en las unidades de producción de la región. Lamentablemente, en México hasta el momento no se ha evaluado la capacidad que tienen dichas bacterias causantes de mastitis bovina, para formar biofilms; y su posible relación con los niveles de resistencia a antibióticos. Con base en lo anterior, el objetivo del presente trabajo fue analizar si existe correlación entre el perfil de resistencia a antibióticos y la formación de biofilm de los aislados de $S$. aureus provenientes de mastitis bovina.

\section{Material y métodos}

\section{Asilamiento de S. aureus}

Se analizaron 30 aislados de $S$. aureus procedentes de casos de mastitis subclínica de vacas localizadas en granjas lecheras ubicadas en localidades de los estados de Guanajuato y Michoacán, México, las cuales utilizan sistemas de producción semi-intensivo y de traspatio. El muestreo, aislamiento y caracterización de los aislados ya fue reportado por Varela et al en $2018^{(12)}$. 


\section{Perfil de multirresistencia a antibióticos}

Se realizó un antibiograma por el método de difusión en disco Kirbi Bauer ${ }^{(13)}$, utilizando sensidiscos de la marca Biorad ${ }^{\circledR}$ con los siguientes antibióticos y concentraciones: penicilina (PE) $6 \mu \mathrm{g}$, oxacilina (Oxa) $6 \mu \mathrm{g}$, dicloxacilina (DC) $30 \mu \mathrm{g}$, pefloxacina (PEF) $5 \mu \mathrm{g}$, cefuroxima (CXM) $30 \mu \mathrm{g}$, gentamicina (GE) $120 \mu \mathrm{g}$, cefotaxima (CTX) $30 \mu \mathrm{g}$, sulfametoxazol + trimetoprima (SXT) 1.25 y $23,75 \mu \mathrm{g}$, tetraciclina (TE) $30 \mu \mathrm{g}$, ampicilina (AM) $10 \mu \mathrm{g}$, eritromicina (E) $15 \mu \mathrm{g}$, ceftazidima (CAZ) $30 \mu \mathrm{g}$ y cefalotina (CF) $30 \mu \mathrm{g}$. Los resultados se reportan como sensible, intermedio y resistente con base en los parámetros establecidos en Performance Standards for Antimicrobial Susceptibility Testing, 2019 $9^{(14)}$. Una vez establecido el perfil de resistencia, los 30 aislados se clasificaron como se describe a continuación: Grupo 1: Resistencia alta (resistentes a 11-13 antibióticos), Grupo 2: Resistencia media (9-10 antibióticos), Grupo 3: Resistencia baja (4-8 antibióticos).

\section{Formación de biofilm}

Para medir la capacidad formadora de biofilm de los aislados de $S$. aureus, se utilizó el protocolo de tinción con cristal violeta ${ }^{(11)}$, como se describe a continuación: se cultivó el aislado bacteriano en medio LB y se incubó $24 \mathrm{~h}$ a $37^{\circ} \mathrm{C}$. Los ensayos se realizaron en placas estériles de 96 pozos y se colocaron $\approx 1 \times 10^{6}$ UFC en un volumen final de $100 \mu \mathrm{l}$ en cada pozo. Los aislados se incubaron 48 horas a $37^{\circ} \mathrm{C}$.

Una vez transcurrido el tiempo de incubación, se descartó el sobrenadante, se lavaron los pozos con $100 \mu \mathrm{l}$ de solución PBS $\left(137 \mathrm{mM} \mathrm{NaCl}, 2.7 \mathrm{mM} \mathrm{KCl}, 8 \mathrm{mM} \mathrm{Na}_{2} \mathrm{HPO}_{4}\right.$ y $2 \mathrm{mM}$ $\mathrm{KH}_{2} \mathrm{PO}_{4}$ ) y se secaron los pozos. Posteriormente, a cada pozo se adicionaron $100 \mu \mathrm{lde}$ solución de cristal violeta ( $0.5 \%$ peso/volumen) y se dejó reposar $15 \mathrm{~min}$. Después se eliminó el colorante y se lavó dos veces con $100 \mu \mathrm{l}$ de PBS. Después se adicionaron $125 \mu \mathrm{l}$ de etanol al 95\% (volumen/volumen) y se resuspendió vigorosamente para disolver el colorante. Se tomó la lectura de la absorbancia a $495 \mathrm{~nm}$ en un analizador de microelisa ELIREAD (Kontrolab $^{\circledR}$, Guidonia, Italia). Una vez obtenidos los datos, se graficó el porcentaje de formación de biofilm utilizando como $100 \%$ la absorbancia registrada de la cepa certificada de $S$. aureus (ATCC 27543). Se realizaron tres experimentos independientes con tres repeticiones.

\section{Análisis de los genes de resistencia y formación de biofilm}

La presencia de genes relacionados con la resistencia a antibióticos y formación de biofilm se realizó a partir del ADN genómico, el cual se obtuvo picando una colonia bacteriana de una placa de cultivo fresco, para posteriormente colocarla en la mezcla de reacción de PCR. Los oligonucleótidos utilizados en este estudio se muestran en el Cuadro 1. La reacción se 
realizó en un volumen final de $20 \mu \mathrm{l}$ que contenía oligonucleótidos $0.4 \mu \mathrm{M}$, desoxinucleótidos trifosfatos $200 \mu \mathrm{M}$ (Invitrogen, Carlsbad, California, Estados Unidos), cloruro de magnesio $2 \mathrm{mM}$ (Invitrogen, Invitrogen, Carlsbad, California, Estados Unidos) y $1 \mathrm{U}$ de Taq polimerasa (Invitrogen, Invitrogen, Carlsbad, California, Estados Unidos). Las condiciones de amplificación fueron las siguientes: temperatura de desnaturalización inicial a $95{ }^{\circ} \mathrm{C}$ durante $10 \mathrm{~min}$, seguida de 30 ciclos de amplificación de desnaturalización durante 10 min a $94{ }^{\circ} \mathrm{C}$, alineamiento durante $1 \mathrm{~min}$ a la temperatura específica de los oligonucleótidos (Cuadro 1), polimerización durante $30 \mathrm{seg}$ a $72{ }^{\circ} \mathrm{C}$, y un ciclo de extensión final durante $7 \mathrm{~min}$ a $72{ }^{\circ} \mathrm{C}$. Los amplicones $(5 \mu \mathrm{l})$ se analizaron por electroforesis en un gel de agarosa al $1 \%$ (peso/volumen) y se tiñeron con bromuro de etidio. Se consideró positivo para el gen, la presencia de una banda de amplificación correspondiente al tamaño del producto esperado.

Cuadro 1: Oligonucleótidos (OLIG) utilizados

\begin{tabular}{|c|c|c|c|c|}
\hline OLIG & Secuencia & $\mathbf{T A}\left({ }^{\circ} \mathbf{C}\right)$ & $\begin{array}{r}\text { TPE } \\
(\mathbf{p b})\end{array}$ & Referencia \\
\hline blaZ & $\begin{array}{l}5^{\prime} \text {-TAAGAGATTTGCCTATGCTT-3' } \\
\text { 5'-TTAAAGTCTTACCGAAAGCAG-3' }^{\prime}\end{array}$ & 49 & 377 & $\begin{array}{l}\text { Yang et al., } \\
2016^{(29)}\end{array}$ \\
\hline mecA & 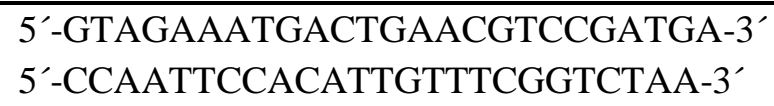 & 62 & 310 & $\begin{array}{l}\text { Elhassan et } \\
\text { al., } 2015^{(27)}\end{array}$ \\
\hline tet $K$ & $\begin{array}{l}\text { 5'-GTAGCGACAATAGGTAATAGT-3' } \\
\text { 5'-GTAGTGACAATAAACCTCCTA-3' }\end{array}$ & 49 & 360 & $\begin{array}{l}\text { Yang et al., } \\
2016^{(29)}\end{array}$ \\
\hline tetM & $\begin{array}{l}5^{\prime} \text {-AGTGGAGCGATTACAGAA-3' } \\
\text { 5'-CATATGTCCTGGCGTGTCTA-3' }\end{array}$ & 49 & 158 & $\begin{array}{l}\text { Yang et al., } \\
2016^{(29)}\end{array}$ \\
\hline gyrA & $\begin{array}{l}5^{\prime} \text {-AATGAACAAGGTATGACACC-3' } \\
5^{\prime} \text {-ACGCGCTTCAGTATAACGC-3' }\end{array}$ & 49 & 222 & $\begin{array}{l}\text { Hashem et } \\
\text { al., 2013 }\end{array}$ \\
\hline gyrB & $\begin{array}{l}\text { 5'-CAGCGTTAGATGTAGCAAGC-3' } \\
\text { 5'-CCGATTCCTGTACCAAATGC-3' }\end{array}$ & 49 & 250 & $\begin{array}{l}\text { Hashem et } \\
\text { al., } 2013^{(28)}\end{array}$ \\
\hline$i c a A$ & $\begin{array}{l}\text { 5'-CCTAACTAACGAAAGGTAG-3' } \\
\text { 5'-AAGATATAGCGATAAGTGC-3' }\end{array}$ & 50 & 1315 & $\begin{array}{l}\text { Dhanawade, } \\
2010^{(49)}\end{array}$ \\
\hline$i c a D$ & $\begin{array}{l}\text { 5'-AAACGTAAGAGAGGTGG-3' } \\
\text { 5'-GGCAATATGATCAAGATAC-3' }\end{array}$ & 50 & 381 & $\begin{array}{l}\text { Dhanawade, } \\
2010^{(49)}\end{array}$ \\
\hline nuc & 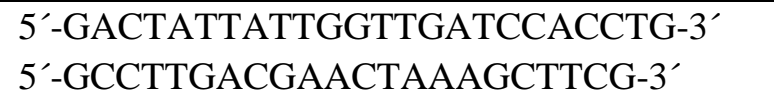 & 54 & 218 & $\begin{array}{l}\text { Brakstad et } \\
\text { al., } 2002^{(50)}\end{array}$ \\
\hline
\end{tabular}

$\mathrm{TA}=$ temperatura de alineamiento; TPE= tamaño del producto esperado.

Para analizar el fundamento genético de los mecanismos de resistencia bacteriana, se analizó la presencia de los genes blaZ y mecA para los antibióticos betalactámicos ${ }^{(15,16)}$; tet $K \mathrm{y}$, tetM para tetraciclinas $^{(16)}$ y gyrA y $_{\text {gyrB }}$ para quinolonas ${ }^{(17)}$. 


\section{Análisis estadístico}

Se realizaron tres experimentos independientes en los cuales se midió la absorbancia producida por la tinción del biofilm formado. Los experimentos fueron hechos por triplicado. Se obtuvo la diferencia entre la mayor absorbancia, menos la mayor cantidad de biofilm, dicha diferencia definida en este estudio como absorbancia.

Los 30 aislados de $S$. aureus se clasificaron en tres grupos de acuerdo con el nivel de resistencia a antibióticos: alta, media y baja, de 10 aislados en cada uno y posteriormente fueron evaluados de acuerdo con su absorbancia. En los análisis se incluyeron los resultados de los controles positivos para cada nivel de resistencia de cada experimento.

Se evaluó la normalidad de la variable dependiente absorbancia, utilizando la prueba de bondad de ajuste de Ji cuadrada resultando normal $(P>0.05)$. Los datos fueron analizados con un análisis de varianza (ANDEVA) con un diseño factorial con arreglo completamente al azar. El modelo utilizado se muestra a continuación:

Yijk $=\mu+E X i+G R j+E X i x G R j+e i j k$, en donde:

Yijk= es la k-ésima observación de absorbancia, del i-ésimo experimento y el j-ésimo grado de resistencia,

$\boldsymbol{\mu}=$ media general como parámetro constante,

$\mathbf{E X i}=$ i-ésimo experimento, $\mathrm{j}=1,2$ y 3 ,

$\mathbf{G R} \mathbf{j}=\mathbf{j}$-ésimo grado de resistencia, $\mathrm{i}=1,2$ y 3 ,

GRixEXj= interacción entre el i-ésimo grado de resistencia y el j-ésimo experimento, eijk= error experimental.

Adicionalmente, se estimó la correlación de rango de Spearman entre grado de resistencia y la absorbancia.

\section{Resultados y discusión}

\section{Perfil de resistencia a antibióticos de los aislados de $S$. aureus provenientes de mastitis bovina}

Los resultados muestran que el $100 \%$ de los aislados son resistentes a la penicilina y dicloxacilina, además el 86.6, 83.3 y $80.0 \%$ muestran resistencia a la ampicilina, cefalotina

y ceftazidima, respectivamente; se observó también que un $36.6 \%$ de los aislados son resistencia a la oxacilina. En general, todos los aislados analizados fueron resistentes al menos al $33.3 \%$ de los antibióticos probados (Cuadro 2). 
Cuadro 2: Perfil de resistencia de los aislados de S. aureus

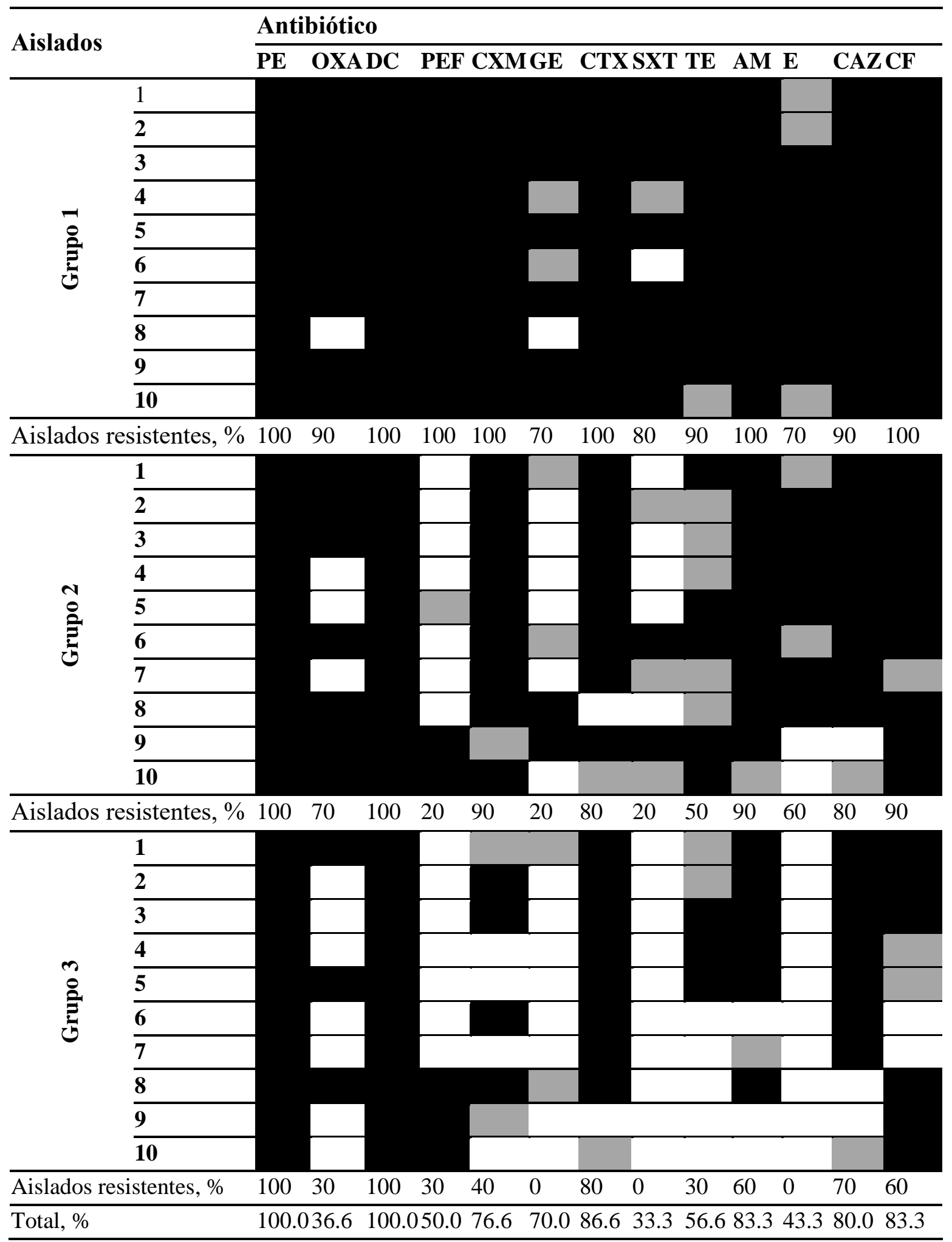

Código de color: Negro, aislado resistente; gris, aislado con resistencia intermedia; blanco, aislado sensible. 
La resistencia a antibióticos es un fenómeno que sigue en aumento y que afecta de manera importante al sector salud, tanto en la medicina humana como en la medicina veterinaria, ya que dificulta el manejo adecuado de las enfermedades infecciosas. Tal es el caso de la mastitis bovina; S. aureus, como una de las principales bacterias aisladas de mastitis bovina presenta altos índices de resistencia. En los últimos años, a nivel mundial, continúa en aumento la selección de mecanismos de resistencia bacteriana ${ }^{(18)}$. En este sentido, en diversos países donde se analizó el perfil de resistencia de $S$. aureus proveniente de mastitis bovina, se encontraron porcentajes de resistencia a penicilina cercanos al $100 \%{ }^{(19-23)}$, lo que coincide con los resultados aquí reportados. Con relación a la resistencia presentada a la oxacilina, se encontraron resultados contrastantes, ya que estudios realizados en países como la India y en China se presentaron niveles de resistencia del 48 al $84 \%{ }^{(21,24)}$; sin embargo, en Alemania, Japón y Colombia los niveles de resistencia a oxacilina son mínimos $(2-7 \%)^{(21,23,25)}$, mientras que estos resultados muestran niveles intermedios de resistencia (33.3 \%). En México, estudios revelan elevados niveles de resistencia bacteriana a penicilina, amoxicilina y dicloxacilina $(100 \%)^{(26,27)}$, lo que es congruente con lo reportado en este trabajo. Asimismo, existe un incremento importante en la resistencia a la cefalotina, por ejemplo, en el 2008 se reportó que el $30 \%$ de las cepas de $S$. aureus estudiadas presentaban resistencia ${ }^{(27)}$; sin embargo, en este trabajo se encontró resistencia de hasta $83 \%$. Estas variaciones pueden deberse a la posible variabilidad genética de los aislados, a las diferencias climatológicas, así como discrepancias geográficas entre otros factores ${ }^{(28)}$. Con base en estas diferencias, se resalta la necesidad de realizar trabajos como el que aquí se expone, para definir las características de virulencia de los S. aureus aislados de regiones particulares; para generar la información necesaria que permita la implementación de tratamientos más eficientes para la mastitis bovina subclínica.

La presencia del gen blaZ fue observada en el $100 \%$ de los aislados analizados, mientras que sólo el $36.6 \%$ resultó positivo para el gen mecA (Cuadro 3). Estos resultados concuerdan con lo reportado con anterioridad, donde se mostró la presencia de estos genes en la totalidad de los aislados analizados ${ }^{(19,29)}$. La presencia de los genes tet $K$, tet $M$, gyrA y gyr $B$ fue expresada de manera descendiente en función de los grupos analizados, lo cual es congruente con el fenotipo encontrado en los antibiogramas. Esta coherencia entre el fenotipo y el genotipo de resistencia bacteriana ya ha sido demostrada con anterioridad ${ }^{(29,30)}$, por lo que la resistencia observada se puede atribuir a la presencia y eventual expresión de los genes analizados ${ }^{(31)}$. 
Cuadro 3: Análisis de genes de resistencia y formación de biofilm

\begin{tabular}{|c|c|c|c|c|c|c|c|c|c|}
\hline \multirow{2}{*}{\multicolumn{2}{|c|}{ Aislados }} & \multicolumn{6}{|c|}{ Genes de resistencia } & \multicolumn{2}{|c|}{ Biofilm } \\
\hline & & blaZ & mect & tet $K$ & tetM & gyr $A$ & $\operatorname{gyr} B$ & icaA & icaD \\
\hline \multirow{10}{*}{$\begin{array}{l}\overrightarrow{0} \\
\stackrel{2}{7}\end{array}$} & 1 & + & + & t & t+ & + & - & t & + \\
\hline & 2 & + & + & + & - & + & + & + & + \\
\hline & 3 & + & + & - & + & t & - & + & + \\
\hline & 4 & + & + & - & - & + & - & + & + \\
\hline & 5 & + & - & + & + & + & + & - & + \\
\hline & 6 & + & + & - & - & + & + & + & + \\
\hline & 7 & + & + & - & + & + & + & + & - \\
\hline & 8 & + & - & + & + & + & + & + & + \\
\hline & 9 & + & + & - & t & + & + & t & + \\
\hline & 10 & + & + & - & + & + & + & + & + \\
\hline \multicolumn{2}{|c|}{ Presencia del gen $(\%)$} & 100 & 80 & 40 & 70 & 100 & 70 & 90 & 90 \\
\hline \multirow{10}{*}{ 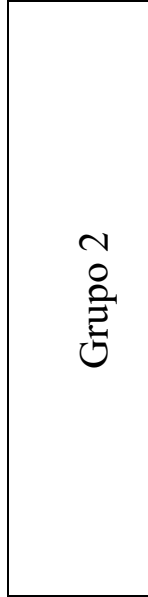 } & 1 & + & + & + & - & - & + & + & + \\
\hline & 2 & + & - & + & - & - & - & + & + \\
\hline & 3 & + & - & - & + & - & + & + & - \\
\hline & 4 & + & - & + & - & - & - & + & + \\
\hline & 5 & + & + & + & + & + & - & + & - \\
\hline & 6 & + & + & + & + & - & - & + & + \\
\hline & 7 & + & - & + & - & - & - & + & + \\
\hline & 8 & + & - & + & - & - & - & - & + \\
\hline & 9 & + & + & + & - & + & + & - & + \\
\hline & 10 & + & + & - & + & + & + & + & + \\
\hline \multicolumn{2}{|c|}{ Presencia del gen (\%) } & 100 & 50 & 80 & 40 & 30 & 40 & 80 & 80 \\
\hline \multirow{10}{*}{$\begin{array}{l}m \\
\stackrel{0}{0} \\
\stackrel{2}{\Xi}\end{array}$} & 1 & + & - & + & - & - & - & + & + \\
\hline & 2 & + & - & - & t+ & - & - & + & - \\
\hline & 3 & + & - & + & + & - & - & + & + \\
\hline & 4 & + & - & + & + & - & - & + & + \\
\hline & 5 & + & + & + & + & - & + & + & + \\
\hline & 6 & + & - & - & + & - & - & + & + \\
\hline & 7 & + & - & - & - & - & - & - & + \\
\hline & 8 & + & - & - & - & + & + & + & + \\
\hline & 9 & + & - & - & - & - & + & + & + \\
\hline & 10 & + & - & - & - & + & + & + & + \\
\hline \multicolumn{2}{|c|}{ Presencia del gen, $\%$} & 100 & 10 & 40 & 50 & 20 & 40 & 90 & 90 \\
\hline \multicolumn{2}{|l|}{ Total, $\%$} & 100.0 & 46.6 & 53.3 & 53.3 & 50.0 & 50.0 & 86.6 & 86.6 \\
\hline
\end{tabular}




\section{Formación de biofilm de $S$. aureus aislado de mastitis bovina}

El análisis de la formación de biofilm mostró que la totalidad de los aislados presentaron la capacidad de formar biofilm con un rango del 20 a $98 \%$. Diversos estudios alrededor del mundo reportan que las cepas de $S$. aureus analizadas presentan la capacidad de formación de biofilm (90 a $99 \%)^{(32,33)}$, lo que coincide con el presente estudio.

Debido a que $S$. aureus presenta altos niveles de resistencia a antibióticos; es necesario analizar los factores y características de virulencia que posee esta bacteria para poder diseñar estrategias de control más eficientes. En este sentido, ya se ha reportado que $S$. aureus tiene la capacidad de formar biofilm ${ }^{(34)}$, lo cual puede estar relacionado con la baja efectividad que presentan los fármacos utilizados convencionalmente ${ }^{(35)}$.

Para establecer la posible relación entre la resistencia y la formación de biofilm en los 30 aislados provenientes de mastitis bovina, se siguió la siguiente estrategia. Primeramente, los resultados de formación de biofilm se analizaron en función del nivel de resistencia de los aislados (Figura 1); para lo cual, estos fueron ordenados en tres grupos como se describe en la sección de materiales y métodos. En el análisis de varianza de este estudio, solo el efecto de grado de resistencia resultó significativo $(P<0.01)$, encontrando que los niveles promedio de absorbancia de los aislados fueron de 1.34 (63.17 \%), 0.77 (38.78 \%) y $0.66(26.28 \%)$ para los grupos 1, 2 y 3, respectivamente. En la comparación de medias, los grupos 1-3 fueron diferentes $(P<0.05)$ (Figura 1B). La correlación estimada entre el grado de resistencia y la formación de biofilm fue positiva $(0.50)$ y significativa $(P<0.01)$ (Figura 2$)$; además, estas diferencias fueron analizadas microscópicamente, donde la formación de biofilm en los aislados del grupo 1 se observaron incrementadas de manera importante en comparación con los otros dos grupos (Figura 1A). Debido a que la formación de biofilm por S. aureus induce al desarrollo de infecciones crónicas y recalcitrantes ${ }^{(32)}$, resalta la necesidad de analizar cómo es que esta característica de virulencia se relaciona con otros mecanismos como la resistencia a antibióticos. Con referencia a lo anterior, esta relación ha sido estudiada tanto en bacterias Gramnegativas ${ }^{(36-39)}$ como Grampositivas ${ }^{(40,41)}{ }^{(4), 3}$ sin embargo, existen discrepancias al definir si la correlación que se presenta es positiva ${ }^{(42,43,44)}$ o negativa ${ }^{(41)}$. En este estudio, se observó que los aislados con una multirresistencia elevada presentan una mayor formación de biofilm; estableciéndose una correlación positiva, la cual es consistente con la mayoría de los trabajos reportados al respecto ${ }^{(42,43,44)}$. Finalmente, se analizó la presencia de los genes icaA, icaD, los cuales se encuentran directamente relacionados con la formación de biofilm ${ }^{(45)}$. Interesantemente, se encontró que existe una alta frecuencia de ambos genes en los aislados (86.6\%), sin importar el grado de resistencia que presentan (Cuadro 3). Diversos autores coinciden con este resultado, ya que reportan una frecuencia cercana al $100 \%$ de uno o ambos genes en las cepas de $S$. aureus formadoras de biofilm ${ }^{(46,47,48)}$. 
Figura 1: Formación de biofilm por los aislados de S. aureus. Para medir la formación de biofilm se utilizó el protocolo de tinción con cristal violeta.

A)

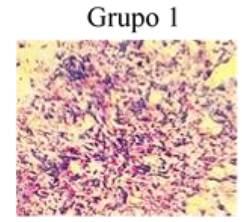

Grupo 2

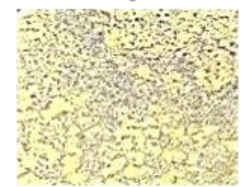

Grupo 3

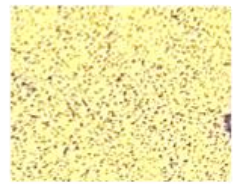

B)

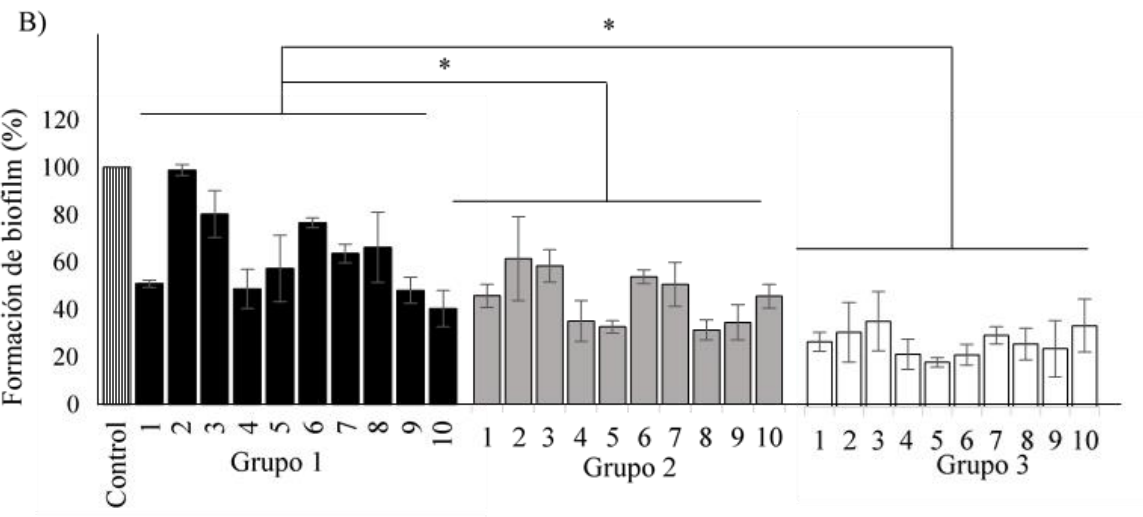

A) Imágenes representativas de la tinción del biofilm en los tres grupos (Grupo 1: Resistencia alta, de 11 a 13 antibióticos; Grupo 2: Resistencia media, de 9 a 10 antibióticos y Grupo 3: Resistencia baja de 4-8 antibióticos), visualizadas con microscopia de campo claro.

B) El gráfico representa el porcentaje de formación de biofilm de los tres grupos de aislados. Se utilizó la cepa certificada de S. aureus ATCC 27543 como control positivo, cuyo valor de absorbancia se estandarizó como el $100 \%$. Se presenta el promedio de tres experimentos independientes por triplicado \pm su desviación estándar. $(*)$ representa diferencia estadísticamente significativa entre grupos $(P \leq 0.001)$.

Figura 2: Relación entre el porcentaje de resistencia y formación de biofilm (\%) de los aislados de $S$. aureus

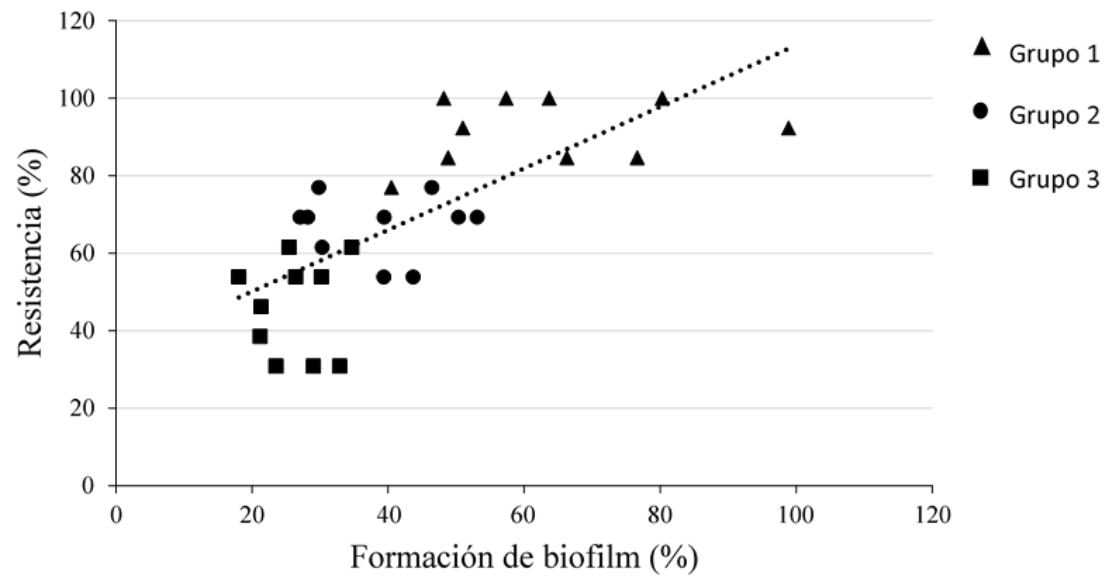




\section{Conclusiones e implicaciones}

Los aislados de $S$. aureus provenientes de mastitis bovina de los estados de Guanajuato y Michoacán, México, presentan elevados niveles de resistencia a antibióticos; así como una importante capacidad formadora de biofilm. Además, en el presente trabajo se demostró la existencia de una relación positiva entre estos dos factores. Estas características de virulencia pueden estar asociadas directamente con el bajo índice de eficacia clínica de los tratamientos utilizados de manera convencional en las granjas lecheras. La variabilidad de los resultados registrados en este estudio y otros reportes en diversas partes del mundo, ponen en evidencia la necesidad de realizar investigaciones sobre las características de virulencia de los microorganismos localizados en una ubicación geográfica específica y con ello establecer estrategias de manejo de la mastitis bovina de una manera integral y eficiente.

\section{Agradecimientos}

La presente investigación fue financiada por la Universidad de Guanajuato a través de la Convocatoria 2018 (DAIP-CIIC-077/2018) y por La Secretaría de Innovación, Ciencia y Educación Superior (MA-CFINN1042).

\section{Literatura citada:}

1. SIAP. Servicio de Información Agroalimentaria y Pesquera. Boletín de Leche. México. 2018.

2. Smith PB. Medicina interna de grandes animales. Serrales, DC (trad.). 4ta ed. Barcelona, España: Elsevier; 2010.

3. Taponen S, Liski E, Heikkilä AM, Pyörälä S. Factors associated with intramammary infection in dairy cows caused by coagulase-negative Staphylococci, Staphylococcus aureus, Streptococcus uberis, Streptococcus dysgalactiae, Corynebacterium bovis, or Escherichia coli. J Dairy Sci 2017;100(1):493-503.

4. Martínez-Sigales JM. Patología y clínica bovina recopilación de clases y relatos de la experiencia práctica de un veterinario de campo. Intermedica. Buenos Aires Argentina. 2016.

5. Gomes F, Henriques M. Control of bovine mastitis: Old and recent therapeutic approaches. Curr Microbiol 2016;72:377-382.

6. Monistero V, Graber HU, Pollera C, Cremonesi P, Castiglioni B, Bottini E, CeballosMarquez A. et al. Staphylococcus aureus isolates from bovine mastitis in eight countries: Genotypes, detection of genes encoding different toxins and other virulence genes. Toxins 2018;10:1-22. 
7. Dorneles EMS, Fonseca MDAM, Abreu JAP, Lage AP, Brito MAVP, Pereira CR, Brandão HM, Guimarães AS, Heinemann MB. Genetic diversity and antimicrobial resistance in Staphylococcus aureus and coagulase-negative Staphylococcus isolates from bovine mastitis in Minas Gerais, Brazil. Microbiologyopen 2019;8:e736.

8. Thiran E, Di Ciccio PA, Graber HU, Zanardi E, Ianieri A, Hummerjohann J. Biofilm formation of Staphylococcus aureus dairy isolates representing different genotypes. J Dairy Sci 2018;101:1000-1012.

9. Raza A, Muhammad G, Sharif S, Atta A. Biofilm producing Staphylococcus aureus and bovine mastitis: A review. Mol Microbiol Res 2013;3(1):1-8.

10. Lister JL, Horswill AR. Staphylococcus aureus biofilms: recent developments in biofilm dispersal. Front Cell Infect Microbiol 2014;4:178:1-9.

11. Chen Y, Liu T, Wang K, Hou Ch, Cai S, Huang Y, Du Z, et al. Baicalein Inhibits Staphylococcus aureus biofilm formation and the quorum sensing system in vitro. Plos ONE 2016;11(4):e0153468.

12. Varela-Ortiz DF, Barboza-Corona JE, González-Marrero J, León-Galván MF, ValenciaPosadas M, Lechuga-Arana AA, Sánchez-Felipe CG. Antibiotic susceptibility of Staphylococcus aureus isolated from subclinical bovine mastitis cases and in vitro efficacy of bacteriophage. Vet Res Commun 2018;42:243-250.

13. Hudzicki J. Kirby-Bauer Disk diffusion susceptibility test protocol. Am Soc Microbiology. 2016.

14. CLSI. Performance standards for antimicrobial susceptibility testing. $20^{\text {th }}$ ed. CLSI supplement M100 Wayne, PA: Clinical and Laboratory Standards Institute; 2019.

15. Yang Y, Jiang X, Chai B, Ma L, Li B, Zhang A, Cole JR, Tiedje JM, Zhang T. ARGsOAP: online analysis pipeline for antibiotic resistance genes detection from metagenomic data using an integrated structured ARG-database. Bioinformatics 2016;32(15):2346-2351.

16. Elhassan MM, Ozbak HA, Hemeg HA, Elmekki MA, Ahmed LM. Absence of the mecA gene in methicillin resistant Staphylococcus aureus isolated from different clinical specimens in Shendi City, Sudan. Bio Med Res Int 2015;ID895860:1-5.

17. Hashem RA, Yassin AS, Zedan HH, Amin MA. Fluoroquinolone resistant mechanisms in methicillin-resistant Staphylococcus aureus clinical isolates in Cairo, Egypt J Infect Dev Ctries 2013;7(11):796-803.

18. Chokshi A, Sifri Z, Cennimo D, Horng H. Global contributors to antibiotic resistance. J Glob Infect Dis 2019;11(1):36-42. 
19. Yang F, Zhang S, Shang X, Li H, Zhang H, Cui D, Wang X, et al. Short communication: Detection and molecular characterization of methicillin-resistant Staphylococcus aureus isolated from subclinical bovine mastitis cases in China. J Dairy Sci 2020;103:840-845.

20. Li T, Lu H, Wang X, Gao Q, Dai Y, Shang J, Li M. Molecular characteristics of Staphylococcus aureus causing bovine mastitis between 2014 and 2015. Front Cell Infect Microbiol 2017;7(127):1-10.

21. Cheng J, Qu W, Barkema HW, Nobrega DB, Gao J, Liu G, De Buck J, et al. Antimicrobial resistance profiles of 5 common bovine mastitis pathogens in large Chinese dairy herds. J Dairy Sci 2019;102:1-11.

22. Kadlec K, Entorf M, Peters T. Occurrence and characteristics of livestock-associated methicillin-resistant Staphylococcus aureus in quarter milk samples from dairy cows in Germany. Front Microbiol 2019;10:1295.

23. Thongratsakul S, Usui M, Higuchi H, Takahashi T, Sato T, Poolkhet Ch, Tamura Y. Prevalence and characterization of Staphylococcus aureus isolated in raw milk from cows in Hokkaido, Japan. Trop Anim Healt Prod 2020;52(4):1631-1637.

24. Mistry H, Sharma P, Mahato S, Saravanan R, Kumar PA, Bhandari V. Prevalence and characterization of oxacillin susceptible mecA-positive clinical isolates of Staphylococcus aureus causing bovine mastitis in India. Plos ONE 2016;11(9):e0162256.

25. Jiménez-Velásquez SC, Torres-Higuera LD, Parra-Arango GL, Rodríguez-Bautista JL, García-Castro FE, Patiño-Burbano RE. Perfil de resistencia antimicrobiana en aislamientos de Staphylococcus spp. obtenidos de leche bovina en Colombia. Rev Argent Microbiol 2020;52(2):121-130.

26. León-Galván MF, Barboza-Corona JE, Lechuga-Arana AA, Valencia-Posadas M, Aguayo DD, Cedillo-Pelaez C, Martínez-Ortega EA, Gutierrez-Chavez AJ. Molecular detection and sensitivity to antibiotics and bacteriocins of pathogens isolated from bovine mastitis in family dairy herds of Central Mexico. Bio Med Res Int 2015;2015:615153 1-9.

27. Ochoa-Zarzosa A, Loeza-Lara PD, Torres-Rodríguez F, Loeza-Ángeles H, MascotChiquito N, Sánchez-Baca S, López-Meza JE. Antimicrobial susceptibility and invasive ability of Staphylococcus aureus isolates from mastitis from dairy backyard systems. Antonie van Leeuwenhoek 2008;94:199-206.

28. Karzis J, Petzer IM, Donkin EF, Naidoo V, Etter EMC. Climatic and regional antibiotic resistance patterns of Staphylococcus aureus in South African dairy herds. Onderstepoort J Vet Res 2019;86(1):e1-e9. 
29. Yang F, Wang Q, Wang XR, Wang L, Li XP, Luo JY, Zhang SD, et al. Genetic characterization of antimicrobial resistance in Staphylococcus aureus isolated from bovine mastitis cases in Northwest China. J Integ Agr 2016;15(12):2842-2847.

30. Artursson K, Söderlund R, Liu L, Monecke S, Schelin J. Genotyping of Staphylococcus aureus in bovine mastitis and correlation to phenotypic characteristics. Vet Microbiol 2016;193:156-61.

31. Foster TJ. Antibiotic resistance in Staphylococcus aureus. Current status and future prospects. FEMS Microbiology Reviews 2017;41:430-449.

32. Avila-Novoa MG, Iñíguez-Moreno M, Solís-Velázquez OA, González-Gómez JP, Guerrero-Medina PJ, Gutiérrez-Lomelí M. Biofilm formation by Staphylococcus aureus isolated from food contact surfaces in the dairy industry of Jalisco, Mexico. Hindawi J Food Qual 2018;ID1746139:1-8.

33. Piechota M, Kot B, Frankowska-Maciejewska A, Gruhewska A, Wofniak-Kosek A. Biofilm formation by methicillin-resistant and methicillin-sensitive Staphylococcus aureus strains from hospitalized patients in Poland. BioMed Res Int 2018; ID4657396:17.

34. Archer NK, Mazaitis MJ, Costerton JW, Leid JG, Powers ME, Shirtliff ME. Staphylococcus aureus biofilms properties, regulation and roles in human disease. Virulence 2011;2(5):445-459.

35. Moormeier DE, Bayles KW. Staphylococcus aureus biofilm: A complex developmental organism. Mol Microbiol 2017;104(3):365-376.

36. Dumaru R, Baral R, Shrestha LB. Study of biofilm formation and antibiotic resistance pattern of gram-negative Bacilli among the clinical isolates at BPKIHS, Dharan. BMC Res Notes 2019;12(1):38.

37. Qi L, Li H, Zhang Ch, Liang B, Li J, Wang Li, Du X, et al. Relationship between antibiotic resistance, biofilm formation, and biofilm-specific resistance in Acinetobacter baumannii. Front Microbiol 2016;7:483.

38. Bawankar SK. Correlation of antibiotic resistance and biofilm formation amongst uropathogenic E. coli. Indian J Appl Res 2018;8:4.

39. Cepas V, López Y, Muñoz E, Rolo D, Ardanuy C, Martí S, Xercavins M, et al. Relationship between biofilm formation and antimicrobial resistance in Gram-negative bacteria. Microb Drug Resist 2019;25:1. 
40. Vitale M, Galluzzo P, Bu PB, Carlino E, Spezia O, Alduina R. Comparison of antibiotic resistance profile and biofilm production of Staphylococcus aureus isolates derived from human specimens and animal-derived samples. Antibiotics 2019;8:97.

41. Zhang Y, Xu D, Shi L, Cai R, Li Ch, Yan H. Association between agr type, virulence factors, biofilm formation and antibiotic resistance of Staphylococcus aureus isolates from pork production. Front Microbiol 2018;9:1876.

42. Tahmasebi H, Dehbashi S, Jahantigh M, Arabestani MR. Relationship between biofilm gene expression with antimicrobial resistance pattern and clinical specimen type based on sequence types (STs) of methicillin-resistant $S$. aureus. Mol Biol Rep 2020;47(2):1309-1320.

43. Lin Q, Sun H, Yao K, Cai J, Ren Y, Chi Y. The prevalence, antibiotic resistance, and biofilm formation of Staphylococcus aureus in bulk Ready-to-eat foods. Biomolecules 2019;9:524.

44. Kwon AS, Park GC, Ryuc SY, Limd DH, Lime DY, Choi CL, Park Y, Lima Y. Higher biofilm formation in multidrug-resistant clinical isolates of Staphylococcus aureus. Int J Antimicrob Agents 2008;32:68-72.

45. O'Gara JP. ica and beyond: biofilm mechanisms and regulation in Staphylococcus epidermidis and Staphylococcus aureus. FEMS Microbiol Lett 2007;270:179-188.

46. Castelani L, Pilon LE, Martins T, Rodrigues-Pozzi C, Rodriguez-Pozzi Arcaro J. Investigation of biofilm production and icaA and icaD genes in Staphylococcus aureus isolated from heifers and cows with mastitis. Anim Sci J 2015;86(3):340-4.

47. Gad GFM, El-Feky MH, El-Rehewy MS, Hassan MA, Abolella H, El-Baky RMA. Detection of icaA, icaD genes and biofilm production by Staphylococcus aureus and Staphylococcus epidermidis isolated from urinary tract catheterized patients. J Infect Dev Ctries 2009;3(5):342-351.

48. Kivanc SA, Arik G, Akova-Budak B, Kivanc M. Biofilm forming capacity and antibiotic susceptibility of Staphylococcus spp. with the icaA/icaD/bap genotype isolated from ocular surface of patients with diabetes. Malawi Med J 2018;30 (4):243-249.

49. Dhanawade NB, Kalorey DR, Srinivasan R, Barbuddhe SB, Kurkure NV. Detection of intercellular adhesion genes and biofilm production in Staphylococcus aureus isolated from bovine subclinical mastitis. Vet Res Commun 2010;34:81-89.

50. Brakstad OG, Aasbakk K, Maeland JA. Detection of Staphylococcus aureus by polymerase chain reaction amplification of the nuc gene. $J$ Clin Microbiol 1992;30:1654-1660. 